\title{
EXPOSURE OF CIRCUMFLEX BRANCHES IN THE TILTED, BEATING PORCINE HEART: ECHOCARDIOGRAPHIC EVIDENCE OF RIGHT VENTRICULAR DEFORMATION AND THE EFFECT OF RIGHT OR LEFT HEART BYPASS
}

Paul F. Gründeman, $\mathrm{MD}, \mathrm{PhD}$

Cornelius Borst, MD, PhD

Cees W. J. Verlaan

Huub Meijburg, MD, PhD

Chantal M. Mouës, MD

Erik W. L. Jansen, MD, PhD
Objective: In off-pump coronary surgery, exposure of posterior vessels via sternotomy causes deterioration of cardiac function. Changes in ventricular geometry, valve competence, and hemodynamics after retraction of the beating heart were studied. Subsequently, the modifying effect of right or left heart bypass was investigated. Methods: In six 80-kg pigs, an ultrasound probe was attached to the backside of the left ventricle and the heart was fully retracted with a suction tissue stabilizer. Five pigs underwent additional pump support. Results: During retraction, the right ventricle was squeezed between the pericardium and interventricular septum, thereby decreasing its diastolic cross-sectional area by $62 \% \pm 6 \%(P<.001)$ while, concomitantly, right ventricular end-diastolic pressure increased to $165 \% \pm 19 \%(P=.004)$ of basal values. Stroke volume and mean arterial pressure decreased by $29 \% \pm 6 \%$ and $23 \% \pm 8 \%(P=.007$ and $P=.02$, respectively $)$. Left ventricular shape became somewhat elliptic without changes in preload pressure, and its diastolic cross-sectional area decreased by $20 \% \pm 3 \%(P=.001)$. All valves were competent. Right heart bypass restored left ventricular cross-sectional area, stroke volume, and mean arterial pressure. In contrast, left heart bypass increased blood pressure only marginally. Conclusions: Ninety-degree anterior displacement of the beating porcine heart caused primarily right ventricular dysfunction as a result of mechanical interference with diastolic expansion without concurring valvular incompetence. Right heart bypass normalized stroke volume and mean arterial pressure by increasing left ventricular preload; in contrast, left heart bypass failed to restore systemic circulation. (J Thorac Cardiovasc Surg 1999;118:316-23)
$\mathrm{O}^{\mathrm{s}}$ ff-pump coronary bypass operations through a sternotomy ${ }^{1,2}$ have recently gained renewed interest for multivessel revascularization. ${ }^{3-6}$ Exposing posterior branches by displacing the beating heart (apex points anteriorly), however, tends to decrease arterial pressure considerably, both in the pig model ${ }^{7,8}$ and in patients. ${ }^{5}$ Currently, circulatory deterioration during exposure of

From the Heart Lung Institute, Utrecht University Hospital, Utrecht, The Netherlands.

Received for publication Dec 21, 1998; revisions requested Feb 19, 1999; revisions received March 19, 1999; accepted for publication March 19, 1999.

Address for reprints: Paul F. Gründeman, MD, PhD, Experimental Cardiology Laboratory, Heart Lung Institute, Utrecht University Hospital (Rm G02.523), PO Box 85500, 3508 GA Utrecht, The Netherlands.

Copyright (C) 1999 by Mosby, Inc.

0022-5223/99 \$8.00+ $0 \quad \mathbf{1 2 / 1 / 9 8 7 2 7}$ posterior vessels seems to prevent rapid expansion of coronary operations on the beating heart. ${ }^{9}$ Inotropic support, ${ }^{5}$ volume redistribution, or increasing preloads $s^{5,7,8}$ may, in part, mitigate hemodynamic deterioration during posterior wall revascularization. Ninety-degree displacement causes biventricular heart failure characterized by a major drop in stroke volume, despite elevation of right ventricular (RV) preload and unchanged left ventricular (LV) preload. 7,8 Previously, it was found that the decreased pump function is not caused by impaired blood supply to the myocardium in a retracted beating heart. ${ }^{8}$ Visually, the right ventricle looks crumpled and folded on retraction. ${ }^{7,8}$ Pump failure may be worsened by the presence of concomitant valvular incompetence. It is conceivable that cardiac displacement causes strangulation of ventricles, but it is unclear which ventricle is more affected in its (diastolic) pump function by the maneuver. The precise mechanisms that cause deteriora- 


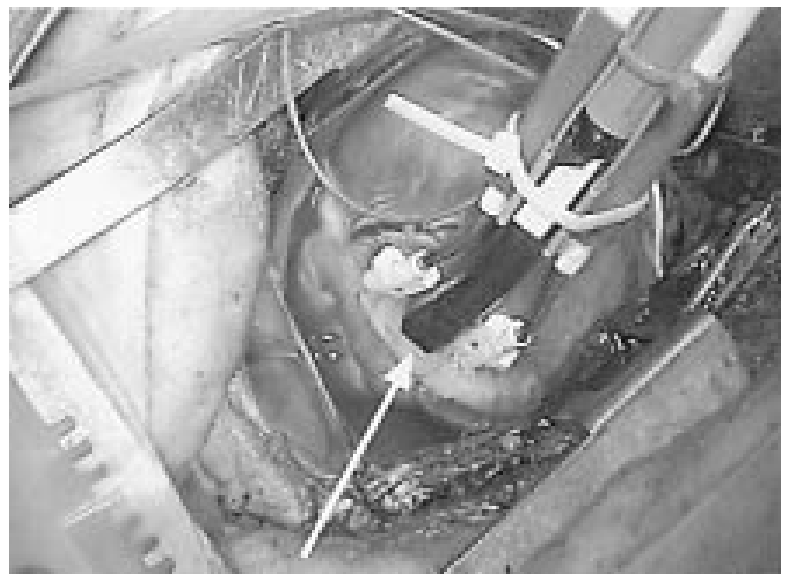

Fig 1. Exposure of posterior vessels by vertical displacement of the beating porcine heart with the Octopus tissue stabilizers, ${ }^{5,10}$ which were mounted to the sternal retractor. Note the omniplane ultrasound probe that was fixed between the suction tentacles (arrow).

tion of the circulation on lifting the beating heart are still not fully understood.

The objectives of study I were (1) to monitor changes in ventricular geometry, hemodynamics, and valvular function during vertical displacement of the beating porcine heart by the Octopus tissue stabilizer (Medtronic, Inc, Minneapolis, Minn $)^{5,7,8,10}$ and (2) to assess the modifying effect of the Trendelenburg maneuver (wholebody head-down positioning) on the changes in ventricular geometry, hemodynamics, and valve function.

In study II we investigated the effect on hemodynamics of mechanically supporting either the right or the left ventricle during vertical displacement of the beating porcine heart.

\section{Methods}

Six Dutch landrace pigs (range 75-85 kg) were used. All animals received humane care in compliance with the "Guide for the Care and Use of Laboratory Animals" published by the National Institutes of Health (NIH publication No. 85-23, revised 1985). The study protocol was approved by the Animal Experimentation Committee of the Utrecht University.

Anesthesia and instrumentation. The animals were premedicated, anesthetized, and instrumented for hemodynamic measurements as described before. ${ }^{8}$ To reduce the mechanical irritability of the heart, we gradually administered intravenous propranolol (range $15-25 \mathrm{mg}$ ) to obtain a heart rate between 50 and 70 beats/min. After median sternotomy, the heart was suspended in a pericardial cradle. A bipolar pacing lead was sutured on the right atrial appendix, whereafter the heart was paced at a fixed rate of 80 beats $/ \mathrm{min}$. An ultrasound transit time flow probe (Transonic Inc, Ithaca, NY, size 20 or

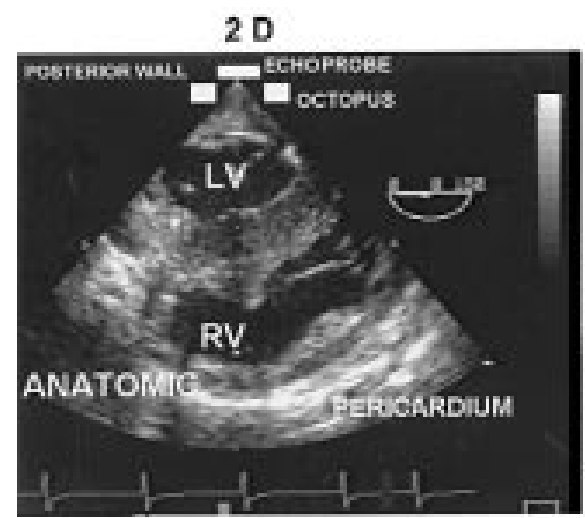

M-MODE

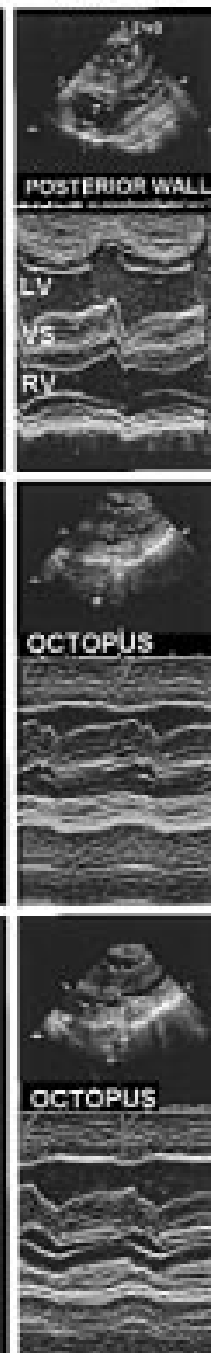

Fig 2. Echographic cross-sectional areas (left panels) and Mmode images (right panels) obtained during 90-degree anterior displacement (DISPLACED) and subsequent head-down positioning (+ TRENDELENBURG). VS, Ventricular septum; $R V$, right ventricle; $L V$, left ventricle. The ultrasound probe was positioned between 2 Octopus tentacles and aligned to the posterior wall (ANATOMIC). Note the severe deformation of the RV on cardiac retraction (arrow, DISPLACED) and the partial recovery of $\mathrm{RV}$ cross-sectional area after the Trendelenburg maneuver.

$24 \mathrm{~mm}$ ) was placed around the aorta for on-line measurement of the cardiac output. Stroke volume was calculated by dividing cardiac output by heart rate (fixed rate $80 / \mathrm{min}$ ). For stepwise cardiac retraction, ${ }^{8}$ two Octopus tissue stabilizers ${ }^{5,10}$ were used, with an omniplane ultrasound probe (6.2. MHz, Sonos 2000, Hewlett-Packard, Amstelveen, The Netherlands) fixed in between (Fig 1). The ultrasound probe was remotely steered to the heart surface until it touched the immobilized 

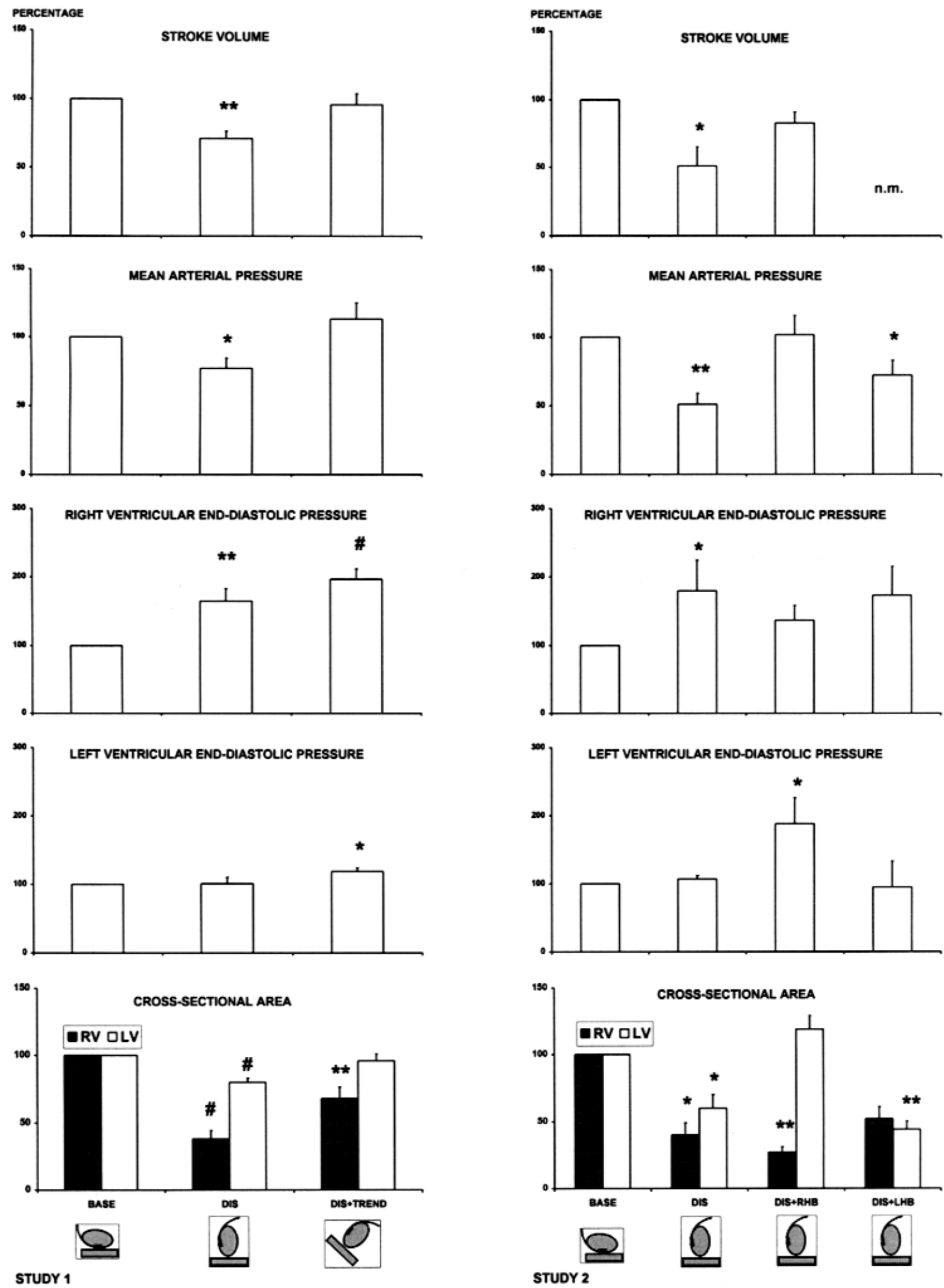

Fig 3. For legend see page 319. 
epicardium for the on-line collection of 2-dimensional and M-mode echocardiographic images (Fig 2). During maneuvers, the ultrasound probe remained exactly positioned in between and in the same plane as the Octopus tentacles. Four 3F air-filled balloon-tipped catheters, which served as echographic beacons, were placed in the lumen of the right ventricle and marked precisely the RV long-axis plane (first pair: RV outflow tract and RV apical portion) or the RV short-axis plane (second pair: border of tricuspid valve and midportion of interventricular grove). Care was taken that the plane of the short-axis view of the left ventricle crossed the apices of both LV papillary muscles.

A personal computer-based data acquisition system stored hemodynamic variables, which were simultaneously monitored on an 8-channel recorder (Gould Instrument System Inc, Valley View, Ohio).

After completion of the experimental protocol of study I (see further), in 5 animals (study II) the right atrium and pulmonary artery trunk and the left atrium and left carotid artery were cannulated for right and left heart bypass (RHB and LHB, respectively), as described before. ${ }^{11}$ A single centrifugal pump (BP 80, Medtronic Bio-Medicus, Eden Prairie, Minn) was connected to the withdrawal and return cannulas by 2 Y-connectors. The pump circuit was used as either RHB or LHB by instantaneously changing tubing clamps. A test run was performed at maximum flow to bypass the right ventricle and the left ventricle (bypass flow $>4 \mathrm{~L} / \mathrm{min}$ ) in the anatomic position. It was assured that the tip of the $38 \mathrm{~F} \mathrm{LHB}$ withdrawal cannula was suspended in the left atrial lumen by applying slight tension to the purse-string suture while the heart was retracted anteriorly. By palpation, the tip position was again verified.

\section{Experimental protocol}

Study I $(n=6)$. Echocardiographic recordings were taken at each phase during at least 5 cardiac cycles and videotaped for analysis. In the same 2-dimensional view (probe at zero degrees), end-diastolic chamber dimensions (square millimeters) were obtained from the short-axis LV cross-sectional area and the long-axis RV cross-sectional area. In the Mmode view (position zero degrees), the distance between the LV posterior wall and RV free wall was measured in millimeters. After 90 degrees' rotation of the ultrasound omniplane probe (probe in position 90 degrees), the RV short-axis cross-sectional area was measured. The LV long-axis view could not be consistantly obtained in the same 2-dimensional plane and was further not measured. In addition, Doppler echocardiographic images were made of the mitral and aortic valves after each maneuver. The function of the pulmonary and tricuspid valves was judged in 2 separate experiments with a hand-held ultrasound Doppler probe surfing over the epicardium using different echographic cross sections. Baseline cardiovascular values and echocardiographic measurements were recorded after fixation of the Octopus tentacles to the posterior wall after at least 15 minutes of pacing (phase 1, anatomic position). Subsequently, values were taken 3 minutes (phases 2-5) and 15 minutes (phases 3 and 5) after stabilization after each intervention.

The heart was vertically displaced by 90 degrees without delay with the Octopus tissue stabilizer (phase 2, first displacement) until the apex pointed upward. Circumflex branches on the back side of the heart became well exposed to the operative field ${ }^{5,7,8}$ without the aid of additional supportive tools. Subsequently, after stabilization of hemodynamics, the operating table was tilted 20 degrees in the headdown position (Trendelenburg maneuver) without changing the position of the heart relative to the body (phase 3). After the Trendelenburg maneuver, the operating table was returned to the horizontal position while the heart remained retracted for 3 minutes (phase 4, second displacement). Subsequently, the heart was put back in the pericardial cradle (phase 5, anatomic position). The entire experimental protocol was repeated after a period of stabilization.

The first 3 consecutive phases in the experimental protocol are schematically depicted on the abcissa of Fig 3.

Study II $(n=5)$. From baseline (anatomic position) the heart was retracted for 3 minutes (displacement). Subsequently, RHB was initiated whereby maximum RHB flow was set to optimally unload the right ventricle. After 3 minutes' stabilization (displacement + RHB), the pump was gradually stopped and the switch was made to LHB. LHB flow was increased until the left atrium was maximally drained and the atrial tissue collapsed (displacement + LHB).

Statistical analysis. First, data sets of 2 subsequently executed protocols from each animal were averaged and mean values were further used for analysis. Single video still frames at end-diastole and mid-systole were grabbed from original tapes and were off-line analyzed by an independent observer (C.M.M.). Data in Tables I and II are presented as mean \pm standard deviation (SD) (absolute values). Hemodynamic variables and echocardiographic variables in the Results section and in Fig 3 are depicted as mean \pm standard error of the mean (SEM) (percentage of basal values). Statistical analysis was performed by multivariate analysis of variance to assess the influence of displacement, and a paired Student $t$ test was used to assess the modifying effect of the Trendelenburg maneuver or RHB/LHB compared with protocol control values.

Fig 3. Relative changes in hemodynamic parameters and RV and LV cross-sectional areas during vertical displacement of the beating porcine heart with the Octopus tissue stabilizer (study I) and the effect of mechanical circulatory support on displacement (study II). Statistical comparison with control values: $* P<.05$, ** $P<.01$, $\# P<.001$. n.m., Not measured; erroneous readings from the ultrasound aortic flow probe caused by turbulent flow from LHB cannula. $D I S$, Displaced; $L V$, left ventricle; $R V$, right ventricle; $R H B$, right heart bypass; $L H B$, left heart bypass. 
Table I. Study I

\begin{tabular}{|c|c|c|c|c|c|c|c|}
\hline & $\begin{array}{l}\text { Basal values } \\
\text { (phase 1) }\end{array}$ & $\begin{array}{c}\text { Vertical } \\
\text { displacement } \\
\text { (phase 2) }\end{array}$ & $\mathrm{P}$ values & $\begin{array}{c}\text { Trendelenburg } \\
\text { 20-degree } \\
\text { head-down } \\
\text { (phase 3) }\end{array}$ & $\mathrm{P}$ values & $\begin{array}{c}\text { Vertical } \\
\text { displacement } \\
\text { (phase 4) }\end{array}$ & $\begin{array}{c}\text { Freely } \\
\text { beating } \\
\text { (phase 5) }\end{array}$ \\
\hline $\mathrm{SV}(\mathrm{mL})$ & $54 \pm 9$ & $37 \pm 7$ & .007 & $50 \pm 7$ & .5 & $37 \pm 7$ & $45 \pm 3$ \\
\hline HR (paced, beats/min) & 80 & 80 & - & 80 & - & 80 & 80 \\
\hline $\mathrm{CO}(\mathrm{L} / \mathrm{min})$ & $4.3 \pm 0.8$ & $3.0 \pm 0.5$ & .007 & $4.0 \pm 0.6$ & .5 & $3.0 \pm 0.6$ & $3.6 \pm 0.2$ \\
\hline MAP (mm Hg) & $66 \pm 14$ & $50 \pm 12$ & .02 & $73 \pm 16$ & .4 & $47 \pm 9$ & $55 \pm 5$ \\
\hline RVEDP (mm Hg) & $6 \pm 2$ & $9 \pm 2$ & .004 & $11 \pm 2$ & .001 & $8 \pm 1$ & $6 \pm 1$ \\
\hline LVEDP (mm Hg) & $10 \pm 2$ & $10 \pm 5$ & .8 & $12 \pm 3$ & .01 & $9 \pm 3$ & $8 \pm 2$ \\
\hline $\mathrm{RAP}(\mathrm{mm} \mathrm{Hg})$ & $7 \pm 2$ & $8 \pm 2$ & .2 & $9 \pm 2$ & .003 & $8 \pm 2$ & $7 \pm 2$ \\
\hline LAP (mm Hg) & $11 \pm 4$ & $9 \pm 3$ & .03 & $10 \pm 2$ & .3 & $9 \pm 3$ & $11 \pm 2$ \\
\hline RVla, diastolic $\left(\mathrm{mm}^{2}\right)$ & $1357 \pm 262$ & $494 \pm 143$ & .001 & $895 \pm 179$ & .01 & $494 \pm 28$ & $1219 \pm 109$ \\
\hline RVsa, diastolic $\left(\mathrm{mm}^{2}\right)$ & $725 \pm 95$ & $287 \pm 79$ & .001 & $412 \pm 55$ & .01 & $254 \pm 5$ & $616 \pm 108$ \\
\hline LVsa, diastolic $\left(\mathrm{mm}^{2}\right)$ & $1317 \pm 90$ & $1050 \pm 127$ & .001 & $1271 \pm 177$ & .5 & $952 \pm 92$ & $1094 \pm 90$ \\
\hline $\begin{array}{l}\text { Distance, LV posterior wall- } \\
\text { RV free wall }(\mathrm{mm})\end{array}$ & $90 \pm 5$ & $75 \pm 5$ & .001 & $82 \pm 5$ & .008 & $75 \pm 5$ & $87 \pm 2$ \\
\hline
\end{tabular}

Values are shown as means \pm standard deviation $(n=6)$. Comparison was made with basal values where appropriate. $S V$, Stroke volume; $H R$, heart rate; $C O$, cardiac output; $M A P$, mean arterial pressure; RVEDP, right ventricular end-diastolic pressure; $L V E D P$, left ventricular end-diastolic pressure; $R V l a$, right ventricular long-axis cross-sectional area; $R V s a$, right ventricular short-axis cross-sectional area; $L V s a$, left ventricular short-axis cross-sectional area.

\section{Results}

All animals survived the entire procedure without our needing to defibrillate or administer inotropic drugs. The paced heart remained in a regular rate of 80 beats/min throughout the experiment. M-mode measurement revealed that the myocardium in between the Octopus tissue stabilizers was well immobilized, and the full-thickness cardiac wall showed almost no wall motion (Fig 2, right panel). The experimental data of studies I and II are summarized in Tables I and II, respectively, and in Fig 3. Data in the text are given as a percentage of baseline values.

Study I. Echocardiography during displacement of the beating heart (Table I, Fig 2, and Fig 3, left panel). Displacement of the beating heart (phase 2) caused the same degree of deterioration of the circulation as observed before. ${ }^{7,8} \mathrm{RV}$ end-diastolic pressure increased to $165 \% \pm 19 \%$ of baseline values. In contrast, no significant change in LV end-diastolic pressure was observed $(101 \% \pm 9 \%)$. Echographically, a significant part of the RV free wall was pressed to the interventricular septum (Fig 2, left panel, displaced), whereas the $\mathrm{RV}$ outflow tract was somewhat narrowed but remained patent. Diastolic dimensions of the long-axis and shortaxis RV cross-sectional areas decreased to $38 \% \pm 6 \%$ and $40 \% \pm 5 \%$, respectively. Diastolic dimensions of the short-axis LV cross-sectional area decreased to $79 \% \pm$ $3 \%$, whereas the LV muscle was shaped elliptically. In M-mode, the distance between the LV posterior wall and RV free wall decreased by $17 \% \pm 2 \%$. Early diastolic LV septal motion was directed toward the RV free wall, thereby further decreasing RV dimension. In no case was incompetence of any valve observed during the entire cardiac cycle. Specifically, the posterior leaflet of the mitral valve did not show prolapse. The 2-dimensional geometry of the cardiac valves seemed grossly unaffected. The superior and inferior caval veins remained fully patent on displacement.

Tilting whole-body head-down 20-degree Trendelenburg position. Three minutes into the Trendelenburg phase (phase 3), stroke volume had increased to $96 \% \pm 8 \%$ and mean arterial pressure had normalized at the expense of increased ventricular preloads. Echocardiographically, a part of the RV free wall was released from the interventricular septum, and the RV outflow tract (in the short-axis view) increased its dimension. Diastolic dimensions of the long-axis and short-axis RV cross-sectional areas increased to $68 \% \pm$ $8 \%$ and $57 \% \pm 1 \%$, respectively. Diastolic short-axis $\mathrm{LV}$ cross-sectional dimension increased to $96 \% \pm 5 \%$, whereas the left ventricle remained shaped elliptically. The distance between the LV posterior wall and RV free wall increased to $92 \% \pm 2 \%$. Again, in no case was dysfunction of any valve observed during the entire cardiac cycle after the preloads were increased.

Return of the operating table to the horizontal position. After the operating table had been returned to the horizontal position (phase 4, the heart remained retracted 90 degrees), most values returned to preTrendelenburg levels.

Return of the beating heart to the anatomic position. Shortly after return to the anatomic position (phase 5), 
Table II. Study II

\begin{tabular}{|c|c|c|c|c|c|c|c|}
\hline & Basal values & $\begin{array}{c}\text { Vertical } \\
\text { displacement }\end{array}$ & $\mathrm{P}$ values & $\begin{array}{l}\text { RHB during } \\
\text { displacement }\end{array}$ & $\mathrm{P}$ values & $\begin{array}{l}\text { LHB during } \\
\text { displacement }\end{array}$ & $\mathrm{P}$ values \\
\hline $\mathrm{SV}(\mathrm{mL})$ & $42 \pm 7$ & $17 \pm 6$ & .02 & $33 \pm 5$ & .09 & - & - \\
\hline HR (beats/min) & $85 \pm 10$ & $81 \pm 9$ & .07 & $93 \pm 10$ & .1 & $94 \pm 10$ & .06 \\
\hline $\mathrm{CO}(\mathrm{L} / \mathrm{min})$ & $3.4 \pm 0.4$ & $1.5 \pm 0.4$ & .007 & $3.0 \pm 0.5$ & 6 & n.m. & - \\
\hline MAP (mm Hg) & $64 \pm 9$ & $32 \pm 6$ & .006 & $64 \pm 13$ & .9 & $46 \pm 13$ & .04 \\
\hline RVEDP (mm Hg) & $6 \pm 2$ & $9 \pm 1$ & .02 & $7 \pm 1$ & .07 & $9 \pm 2$ & .06 \\
\hline LVEDP (mm Hg) & $6 \pm 1$ & $7 \pm 1$ & .07 & $11 \pm 3$ & .04 & $6 \pm 4$ & .8 \\
\hline RAP (mm Hg) & $8 \pm 2$ & $10 \pm 1$ & .007 & $7 \pm 3$ & .5 & $10 \pm 3$ & .07 \\
\hline LAP (mm Hg) & $7 \pm 1$ & $8 \pm 2$ & .4 & $12 \pm 5$ & .1 & $5 \pm 5$ & .4 \\
\hline RVla, diastolic $\left(\mathrm{mm}^{2}\right)$ & $1249 \pm 31$ & $458 \pm 159$ & .01 & $320 \pm 98$ & .003 & $609 \pm 122$ & .2 \\
\hline LVsa, diastolic $\left(\mathrm{mm}^{2}\right)$ & $1155 \pm 12$ & $679 \pm 214$ & .02 & $1376 \pm 298$ & .1 & $506 \pm 104$ & .002 \\
\hline Maximum pump flow (L/min) & & & & $3.1 \pm 0.5$ & .009 & $1.6 \pm 0.5$ & .001 \\
\hline Ratio, pump flow/baseline $\mathrm{CO}$ & & & & 0.91 & & 0.55 & \\
\hline
\end{tabular}

Values are shown as means \pm standard deviation $(n=5)$. Comparison was made with basal values where appropriate. $S V$, Stroke volume; $H R$, heart rate; $C O$, cardiac output; $M A P$, mean arterial pressure; RVEDP, right ventricular end-diastolic pressure; $L V E D P$, left ventricular end-diastolic pressure; $R A P$, right atrial pressure; $L A P$, left atrial pressure; $R V l a$, right ventricular long-axis cross-sectional area; $L V s a$, left ventricular short-axis cross-sectional area; $n . m$., Not measured.

the right ventricle was transiently dilated. Echocardiographically, RV diastolic dimensions were variably enlarged. Long-axis RV cross-sectional area increased from $1357 \pm 262 \mathrm{~mm}^{2}$ to $1756 \pm 273 \mathrm{~mm}^{2}$ (to $130 \% \pm$ $6 \%, P=.001$, vs baseline values). Central hemodynamic status improved rapidly and RV wall motion increased concomitantly. Diastolic RV dimension decreased to baseline values.

Study II. RHB or LHB in the retracted heart (Table II, Fig 3, right panel). The condition of the animal had somewhat deteriorated because of the previously executed protocol of study I, hemodilution, and subsequent cannulation. At 3 minutes' displacement, RHB was started. Average RHB flow reached $100 \%$ of baseline cardiac output, which increased diastolic short-axis LV cross-sectional dimension, LV preload, and cardiac output to baseline values. In contrast, LHB increased mean arterial pressure to only $72 \% \pm 11 \%$ ( $P$ $=.035)$. Frequently, the LHB withdrawal cannula was pressed against the left atrial wall, which forced reduction of LHB flow. In 2 cases, air aspiration to the centrifugal pump occurred. Maximum LHB flow reached only $55 \%$ of baseline cardiac output.

\section{Discussion}

The principal findings of the study were as follows: First, in the supine animal, vertical displacement of the beating heart caused a major distortion in RV geometry but only a moderate change in LV shape. The decrease in stroke volume and in mean arterial pressure and the increase in RV preload coincided with diminished LV and $\mathrm{RV}$ dimensions.

Second, the borderline circulatory status during dis- placement was markedly improved to almost baseline status by blood volume redistribution (Trendelenburg maneuver). At the same time, LV cross-sectional area, but not LV shape, returned to baseline.

Third, cardiac retraction did not cause valvular dysfunction.

Fourth, RHB effectively restored central hemodynamics in the tilted heart, whereas LHB only marginally improved circulatory status.

Epicardial 2-dimensional echocardiography of the beating heart. The immobilized epicardial surface contained a distal circumflex branch, and its presentation by the Octopus tissue stabilizers simulated full exposure for coronary surgery without the use of additional supportive tools (Fig 1).

In the 80-kg pig, 2-dimensional echocardiography of the beating heart proved technically feasible by fixing the ultrasound probe between two Octopus suction pods, which took hold of the posterior side of the heart (Fig 1). The area immobilized by the retractor/ultrasound system comprized approximately a small matchbox area of cardiac tissue that does not affect cardiac function. ${ }^{7}$ Interestingly, cardiac wall motion and systolic wall thickening between the tentacles was almost absent transmurally in the line of measurement of the echo probe. The latter phenomenon was observed immediately after attachment of the suction device. The function of the mitral valve and the aortic valve could be adequately assessed in all experiments by Doppler ultrasound in the LV long-axis/RV short-axis view.

Displacement of the beating heart. The deterioration in circulation during displacement was characterized by severe reduction in stroke volume despite an 
elevated RV preload without a concomitant change of LV preload and with unimpaired valve function. Thus cardiac retraction causes biventricular pump failure. We hypothesized that the function of the thin-walled right ventricle was primarily affected. When the beating heart was retracted anteriorly, RV luminal dimension in two planes decreased by about $60 \%$, and RV geometry was considerably distorted, evidenced by crumpling of the muscle. For a substantial portion, the thin-walled RV muscle was sqeezed between the left ventricle and the surrounding tissue, that is, the pericardial sac and right sternal border. A significant part of the RV free wall endocardium was pressed against the interventricular septum during the entire cardiac cycle. In diastole, the right ventricle did not expand sufficiently. A rim of RV tissue protruded over the interventricular groove, but that part of the ventricle in fact contributed well to RV output. The augmented RV preload corresponded to the absence of torque or compression of the caval veins and/or tricuspid valve dysfunction and/or pulmonary valve dysfunction. Apparently, the RV lumen available for diastolic expansion was reduced, which amounts to an acquired temporary $R V$ hypoplasia.

In contrast to the severe changes in RV geometry, the changes in LV geometry were only moderate. The shape of the left ventricle changed from circular to elliptic, probably because of a thicker, less deformable muscle. LV function, however, also seemed impaired because stroke volume decreased despite unchanged LV preload and left atrial pressure. Load-independent indices of cardiac contractility were not assessed. Propranolol exerts negative inotropic effects on contractility and may worsen heart failure by displacement. Therefore a complete explanation of the changes of cardiac performance cannot be given.

RV support. In study II, we tested the hypothesis that $\mathrm{RV}$ failure is predominant over left heart failure in a retracted heart. In all cases, selectively increasing the preload of the tilted left ventricle by deviating blood around the displaced right ventricle by means of a short-circuit RHB system fully restored LV stroke volume and, as a result, mean arterial pressure. RHB flow in this study reached baseline cardiac output. In contrast to healthy pigs, patients with poor LV function and dilated hearts may not tolerate augmentation of LV preload in deformed tilted hearts (mean LV end-diastolic pressure increased from 6 to $11 \mathrm{~mm} \mathrm{Hg}$ ). These patients may need a form of LV mechanical support as well.

LV support. In contrast to RV support, LV support resulted in only marginal improvement of mean arterial pressure. Maximum LHB flow was comparable with cardiac output in the displaced heart without any form of circulatory support. Attemps to fully restore systemic circulation failed in all cases because blood supply originating from the right ventricle was apparently insufficient. The left atrial wall easily collapsed and yielded a risk of air aspiration (in 2 of 5 cases). We rule out technical problems related to cannulation because the correct position of the tip of the withdrawal cannula was verified by palpation. From these observations we infer that the severe drop in stroke volume during anterior displacement of the beating porcine heart is primarily caused by right heart pump failure.

Effect of 20-degree Trendelenburg positioning. As reported earlier, ${ }^{7,8}$ the borderline circulatory status during displacement markedly improved almost to baseline status by blood volume redistribution (Trendelenburg maneuver). When the subject was tilted head down, the compressed right ventricle was (in part) mechanically pushed open by further augmenting filling pressure, demonstrated by an improved but incomplete recovery of RV dimensions, while the position of the heart relative to the body remained unchanged. At the same time, LV filling pressure increased, LV dimensions returned to baseline, and stroke volume normalized.

In selected patients, the Trendelenburg maneuver effectively mitigates circulatory deterioration on exposure of the posterior wall. ${ }^{5}$ In two thirds of the cases, however, inotropic support remained necessary. ${ }^{3}$

Recent observations by us showed that right oblique positioning of the pig after sternotomy facilitated exposure of the posterior LV wall and also mitigated the sequelae of cardiac retraction on mean arterial pressure.

The beating heart in the anatomic position. As before, ${ }^{7,8}$ we observed transient firm distention of the right ventricle on quickly replacing the heart into its anatomic position. The dilatation disappeared within several minutes and the central hemodynamic status improved. Apparently, the right ventricle needs to adjust after more than 30 minutes of deformation and increased preload.

Limitations. The pig's chest wall geometry differs from that of the human chest wall; that is, it is less barrel-shaped. Cardiac retraction was carried out in the normal healthy pig heart with its apex slightly pointing rightward. Patients with impaired LV function may not tolerate vertical displacement of the beating heart. Therefore inferences made from this experimental study may be applicable to human beings only after careful consideration.

\section{Conclusion}

Ninety degrees' anterior displacement of the beating porcine heart caused primarily RV deformation and 
pump function without signs of valvular incompetence or inflow or outflow obstruction. The displacement prevented normal RV and LV diastolic expansion by pressing the heart against the surrounding tissue. It is inferred that vertical displacement affects mostly RV function because RHB effectively restored LV function whereas LHB failed to restore cardiac output and mean arterial pressure.

We acknowledge the technical contributions of Hans P. van der Brugge (data aquisition), Stefan L. Damen, and Monique de Baar, clinical perfusionist. Rob Stoopman kindly supported us with the echocardiographic equipment (HewlettPackard, Amstelveen, The Netherlands) and gave technical assistance. For assistance in statistical analysis, we thank Joop A. J. Faber, McS, PhD, and colleagues of the Center of Biostatistics, University of Utrecht.

\section{REFERENCES}

1. Buffolo E, Andrade JCS, Branco JNR, Aguiar LF, Teles CA, Gomes WJ. Coronary artery bypass grafting without cardiopulmonary bypass. Ann Thorac Surg 1996;61:63-6.

2. Benetti FJ, Naselli G, Wood M, Geffner L. Direct myocardial revascularization without extracorporeal circulation: experience in 700 patients. Chest 1991;100:312-6.

3. Pfister AJ, Zaki MS, Garcia JM, Mispireta LA, Corso PJ, Qazi AG, et al. Coronary artery bypass without cardiopulmonary bypass. Ann Thorac Surg 1992;54:1085-92.

4. Moshkovitz Y, Lusky A, Mohr R. Coronary artery bypass without cardiopulmonary bypass: analysis of short-term and mid-term outcome in 220 patients. J Thorac Cardiovasc Surg 1995;110: 979-87.

5. Jansen EWL, Borst C, Lahpor JR, Gründeman PF, Eefting FD, Nierich A, et al. Coronary artery bypass grafting without cardiopulmonary bypass using the Octopus method: results in the first 100 patients. J Thorac Cardiovasc Surg 1998;116:60-7.

6. Tasdemir O, Vural KM, Karagoz H, Bayazit K. Coronary artery bypass grafting on the beating heart without the use of extracorporeal circulation: review of 2052 cases. J Thorac Cardiovasc Surg 1998;116:68-73.

7. Gründeman PF, Borst C, van Herwaarden JA, Mansvelt Beck HJ, Jansen EWL. Hemodynamic changes during displacement of the beating heart by the Utrecht Octopus Method. Ann Thorac Surg 1997;63:S88-92.

8. Gründeman PF, Borst C, van Herwaarden JA, Verlaan CWJ, Jansen EWL. Vertical displacement of the beating heart by the Octopus stabilizer: influence on coronary flow. Ann Thorac Surg 1998;65:1348-52.

9. Borst C, Gründeman PF. Minimally invasive coronary artery bypass grafting: an experimental perspective [editorial]. Circulation. 1999;99:1400-3.

10. Borst C, Jansen EWL, Tulleken CAF, Gründeman PF, Mansvelt Beck HJ, van Dongen JWF, et al. Coronary artery bypass grafting without cardiopulmonary bypass and without interruption of native coronary flow using a novel anastomosis site restraining device ('Octopus'). J Am Coll Cardiol 1996;27:1356-64.

11. Gründeman PF, Tukkie R, Moulijn AC, Rudolphy VJ, de Jong JW, Frederiks WM, et al. Short-term biventricular circulatory support in the regionally stunned pig myocardium. Thorac Cardiovasc Surg 1993;41:290-6.

\section{Targeted}

The Journal of Thoracic and Cardiovascular Surgery gives you two tables of contents.

The condensed table of contents tells you at a glance what topics and authors are presented each month. The expanded table of contents gives you a brief abstract of each article. You select only those articles of most interest to you for further reading. 